\title{
Anti-inflammatory Effects of Sanhuang-Siwu-Tang in Lipopolysaccharide-Stimulated RAW264.7 Macrophages and BV2 Microglial Cells
}

\author{
Jing Li, ${ }^{*, a, b}$ Kwan-Woo Kim, ${ }^{a}$ Hyuncheol Oh, ${ }^{a}$ and Youn-Chul Kim ${ }^{*, a}$ \\ ${ }^{a}$ Institute of Pharmaceutical Research and Development, College of Pharmacy, Wonkwang University; Iksan 54538, \\ Korea: and ${ }^{b}$ Department of Pharmacy, Jiujiang University Affiliated Hospital; Jiujiang 332000, China. \\ Received October 29, 2020; accepted February 4, 2021; advance publication released online February 9, 2021
}

\begin{abstract}
Sanhuang-Siwu-Tang (SST), composed of seven medicinal herbs, is a well-known herbal formula used for the treatment of gynecologic diseases. To expand the clinical use of SST, we explored the antiinflammatory or anti-neuroinflammatory effects of SST water extract in lipopolysaccharide-stimulated RAW264.7 macrophages and BV2 microglial cells. According to HPLC analysis, the main components of SST were from Scutellariae Radix, Coptidis Rhizoma, and Paeoniae Radix. SST significantly inhibited proinflammatory mediators including lipopolysaccharide (LPS)-induced production of nitric oxide (NO) and prostaglandin $\mathrm{E}_{2}\left(\mathrm{PGE}_{2}\right)$ as well as protein expression of inducible nitric oxide synthase (iNOS) and cyclooxygenase-2 (COX-2), and the production of interleukin-6 (IL-6) and tumor necrosis factor- $\alpha$ (TNF- $\alpha$ ) in LPS-stimulated RAW264.7 macrophages and BV2 microglial cells. Furthermore, these anti-inflammatory or anti-neuroinflammatory effects of SST were mediated by mitogen-activated protein kinase-related proteins (MAPK) and nuclear factor $\kappa$-light-chain-enhancer of activated B cells (NF- $\kappa$ B)-related proteins. Overall, this study demonstrated that SST is a potential therapeutic formula for the prevention or treatment of inappropriate inflammation, neuroinflammation, or neurodegenerative diseases.
\end{abstract}

Key words Sanhuang-Siwu-Tang; inflammation; neuroinflammation; RAW264.7 macrophage; BV2 microglial cell

\section{INTRODUCTION}

Sanhuang-Siwu-Tang (SST), recorded in Yi zong jin jian, has been widely used for the treatment of various gynecological diseases, including adolescent functional uterine bleeding and dysmenorrhea. ${ }^{1-3)}$ SST consists of seven medicinal herbs: Angelicae Gigantis Radix (Angelica Gigas Nakai, root), Ligusticum chuanxiong Hort (Ligusticum Hort, rhizome), Rehmanniae Radix Preparata (Rehmannia glutinosa Gaertn. DC., rhizome), Paeoniae Radix (Paeonia lactiflora Pall., root), Rhei Radix Et Rhizoma (Rheumofficinale Baill., rhizome), Scutellariae Radix (Scutellaria baicalensis Georgi, root), and Coptidis Rhizoma (Coptis teeta Wall., rhizome). There have been reports about the anti-inflammatory or anti-neuroinflammatory effects of some of the ingredients of SST. Ligusticum chuanxiong Hort has been reported as anti-inflammatory effects referring to its active substance such as tetramethylpyrazine. ${ }^{4)}$ Paeoniflorin from Paeoniae Radix showed its anti-inflammatory effect mediating by the nuclear factor $\kappa$-light-chain-enhancer of activated B cells (NF- $\kappa$ B) pathway. ${ }^{5)}$ Berberine, an active substance in Coptidis Rhizoma, could decrease neuroinflammatory responses and oxidative stress and also alleviate cognitive impairment. ${ }^{6)}$ Baicalein from Scutellariae Radix attenuates neuroinflammatory effects in lipopolysaccharide (LPS)-stimulated BV2 microglial cells. ${ }^{7)}$ However, the anti-inflammatory or anti-neuroinflammatory effects of the SST formula have not yet been studied.

Macrophages are immune cells that participate in various inflammatory diseases such as rheumatoid arthritis, autoimmune, and primary immunodeficiency diseases. ${ }^{8)}$ RAW264.7 macrophages induced by LPS stimulation have been frequently used to study macrophage activation in inflammatory diseases. ${ }^{9)}$ Microglia, a type of primary immune cell considered to be the macrophages in the central nervous system (CNS), play crucial roles in the neuroinflammation process, leading to a variety of neurodegenerative disorders such as Alzheimer's disease and Parkinson's disease. ${ }^{10,11)}$ BV2 microglial cells are immortalized microglial cells used in in vitro studies as neuroinflammation models. ${ }^{12)}$ In the course of inflammatory and neuroinflammatory reactions, both activated macrophages and microglia cells produce pro-inflammatory mediators, including tumor necrosis factor- $\alpha$ (TNF- $\alpha$ ), interleukin-6 (IL-6), cyclooxygenase-2 (COX-2), prostaglandin $\mathrm{E}_{2}\left(\mathrm{PGE}_{2}\right)$, inducible nitric oxide synthase (iNOS), and nitric oxide (NO). ${ }^{13-15)}$ Additionally, these reactions are related to the activation of and mitogenactivated protein kinase (MAPK) and the NF- $k$ B pathways. ${ }^{16,17)}$ Therefore, inhibiting the production of pro-inflammatory mediators is a strategy for the prevention or treatment of inappropriate inflammation or neuroinflammation.

In this study, we evaluated the anti-inflammatory effects of SST and its associated mechanisms in LPS-stimulated RAW264.7 macrophages and BV2 microglial cells and also analyzed the main components of the SST formula.

\section{MATERIALS AND METHODS}

Plant Materials and Extraction The seven crude herbs were purchased from Daerim Korean crude drug store (Kumsan, Korea) in March 2018, and identified by Dr. Kyu-Kwan Jang, Botanical Garden, Wonkwang University (Iksan, Korea). A voucher specimen was deposited at the Herbarium of the College of Pharmacy, Wonkwang University. SST formula 
(75 g) is composed of seven herbs, Angelicae Gigantis Radix (10 g), Ligusticum chuanxiong Hort (10g), Rehmanniae Radix Preparata (10g), Paeoniae Radix (15g), Rhei Radix Et Rhizoma (10g), Scutellariae Radix (10g), and Coptidis Rhizoma $(10 \mathrm{~g})$. SST $(75 \mathrm{~g})$ was extracted in $500 \mathrm{~mL}$ distilled water at $100{ }^{\circ} \mathrm{C}$ twice $(2.5 \mathrm{~h}$ each time) using a heat reflux extractor. The combined solutions were filtered and concentrated under vacuum with heat, and the amount of extract was $29.14 \mathrm{~g}$ (yield: $38.85 \%$ ). The extracts of seven ingredients were prepared respectively, $5 \mathrm{~g}$ for each, in the same way as SST ex- traction. The samples were stored at $4{ }^{\circ} \mathrm{C}$ before analysis.

Chemicals and Reagents Baicalin was from Wako Pure Chemical Industries, Ltd. (Osaka, Japan), and the purity was $\geq 99.0 \%$. HPLC-grade water and acetonitrile were from J.T.Baker (Phillipsburg, NJ, U.S.A.). Glacial acetic acid, analytical reagent grade, was from Junsei (Tokyo, Japan). RPMI Medium 1640 and fetal bovine serum (FBS) were from Gibco BRL Co. (Grand Island, NY, U.S.A.). LPS (Escherichia coli 055:B5, Lot: 046M4045V) and 3-(4,5-dimethylthiazol2-yl)-2,5-diphenyltetrazolium bromide (MTT) were from
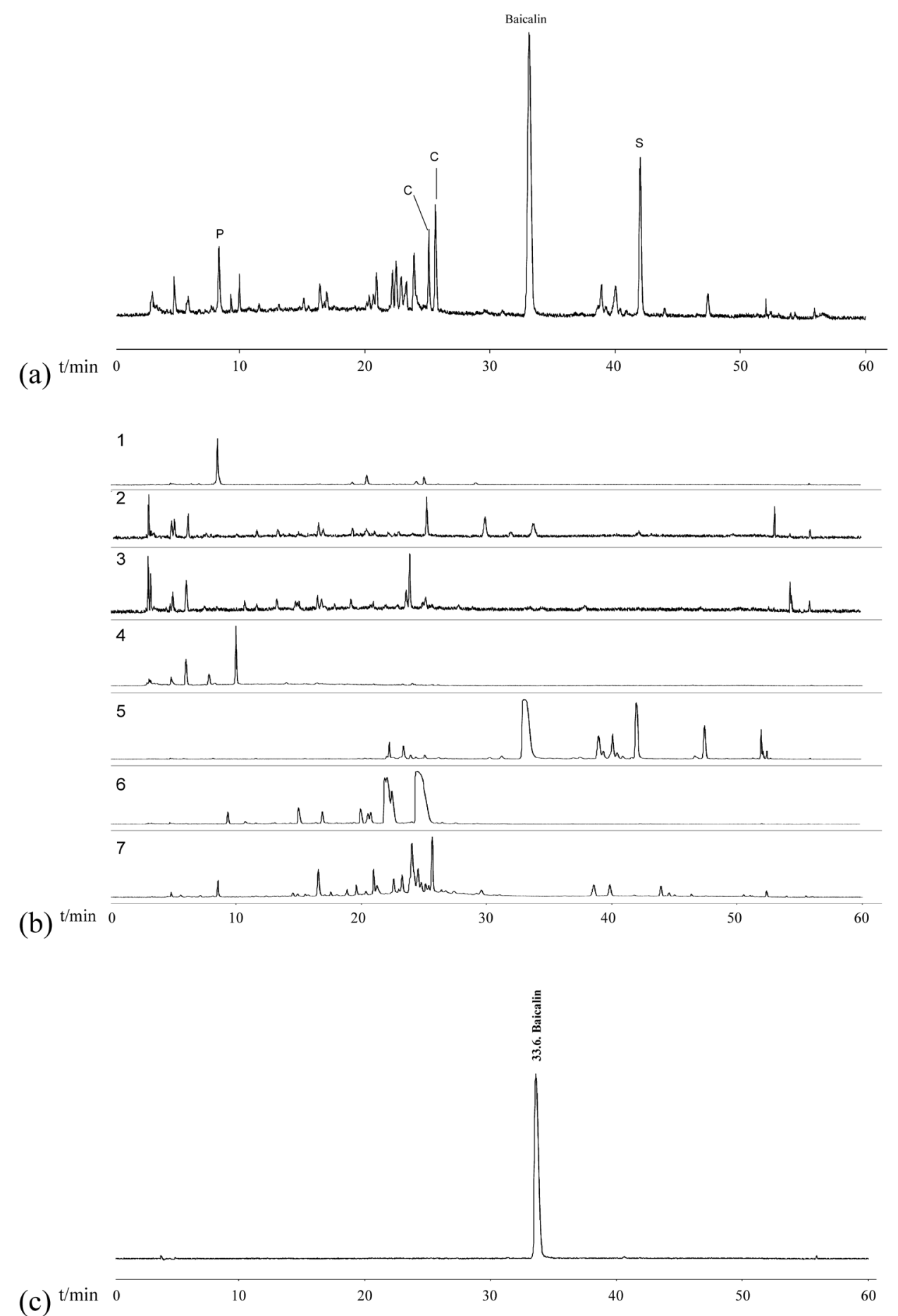

Fig. 1. (a) HPLC Chromatogram of SST, S Represents for Scutellariae Radix, C Represents for Coptidis Rhizoma, and P Represents for Paeoniae Radix; (b) HPLC Chromatogram of Paeoniae Radix [1], Ligusticum Chuanxiong Hort [2], Angelicae Gigantis Radix [3], Rehmanniae Radix Preparata [4], Scutellariae Radix [5], Coptidis Rhizoma [6], and Rhei Radix Et Rhizoma [7]; (c) HPLC Chromatogram of Baicalin 
Sigma-Aldrich (St. Louis, MO, U.S.A.). Primary antibodies including anti-iNOS, anti-COX-2, anti-inhibitor kappa B (I $\kappa \mathrm{B})-\alpha$, anti-p-I $\kappa \mathrm{B}-\alpha$, anti-p65, anti-p-extracellular signalregulated kinase (ERK), anti-ERK, anti-p-c-Jun N-terminal kinase (JNK), anti-JNK, anti-p-p38, anti-p38, anti-proliferating cell nuclear antigen (PCNA), and anti-actin were from Cell Signaling Technology (Danvers, MA, U.S.A.). Secondary antibodies including anti-mouse and anti-rabbit were from Merck Millipore (Darmstadt, Germany). The enzyme-linked immunosorbent assay (ELISA) kit for PGE 2 , IL-6, and TNF- $\alpha$ were from R\&D Systems, Inc. (Minneapolis, MN, U.S.A.). The Nuclear Extraction kit was from Cayman Chemical, Inc. (Ann Arbor, MI, U.S.A.). Radio immunoprecipitation assay (RIPA) buffer and Protease \& Phosphatase Inhibitor Cocktail $(100 \times)$ were from Thermo Fisher Scientific (Waltham, MA, U.S.A.). Enhanced chemiluminescent (ECL) Prime Western blotting Detection Reagent was from GE Healthcare (PA, U.S.A.). Bradford protein assay and nitrocellulose (NC) membranes were from Bio-Rad Laboratories (CA, U.S.A.).

HPLC Analysis The chromatographic analysis was performed using the YL9100 HPLC system (Young Lin Chromass Co., Korea) consisting of YL9101 Vacuum degasser, YL9110 Quaternary pump, and YL9160 PDA detector. The analytes were separated by Phenomenex Gemini NX-C18 $(250 \times 4.6 \mathrm{~mm}, 5 \mu \mathrm{m})$. The data were acquired and processed by software YL-Clarity (Young Lin Instruments Co., Inc., Version 4.0.3.876). The mobile phases consisted of $0.1 \%(\mathrm{v} / \mathrm{v})$ aqueous formic acid in water (A) and acetonitrile (B). The gradient flow was as follows: $0-20 \min 5-25 \% \mathrm{~B}, 20-30 \mathrm{~min} 25 \%$ B, $30-45 \min 25-45 \%$ B, $45-50$ min $45-100 \%$ B, $50-60$ min $100 \%$ B. All the samples were dissolved with distilled water to $10 \mathrm{mg} / \mathrm{mL}$, the injection volume was $10 \mu \mathrm{L}$, the flow-rate was $0.7 \mathrm{~mL} / \mathrm{min}$, and the detection wavelength was $280 \mathrm{~nm}$.

Cell Culture and Viability Assay RAW264.7 macrophages and BV2 microglial cells were from Prof. Hyun Park at Wonkwang University. RAW264.7 macrophages and BV2 microglial cells were cultured in RPMI medium, with $10 \%$ heat-inactivated FBS, penicillin G (100 units/mL), and streptomycin $(100 \mu \mathrm{g} / \mathrm{mL})$, and incubated in humidified atmosphere containing $5 \% \mathrm{CO}_{2}$ at $37^{\circ} \mathrm{C}$. Cell viability was evaluated by MTT assay. Briefly, MTT $(50 \mu \mathrm{L}, 2.5 \mathrm{mg} / \mathrm{mL})$ was added to the cell supernatant and then further incubated for $3 \mathrm{~h}$ at $37^{\circ} \mathrm{C}$. The formazan formed was dissolved in dimethyl sulfoxide (DMSO), and the optical density was measured at $540 \mathrm{~nm}$. The optical density of the formazan formed in the control group was considered as $100 \%$ viability.

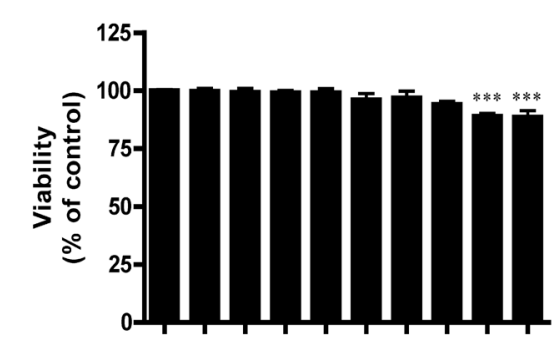

(a) $\operatorname{SST}(\mu \mathrm{g} / \mathrm{mL}) \quad-\quad 0.78 \quad 1.563 .136 .25 \quad 12.5 \quad 25 \quad 50 \quad 100 \quad 200$
Determination of NO, PGE ${ }_{2}$, IL-6, and TNF- $\alpha$ The supernatant was collected for measuring. NO was measured by Griess reaction. PGE 2 , IL- 6 , and TNF- $\alpha$ were detected by ELISA kits according to the manufacturers' instructions. Butein $(10 \mu \mathrm{M})$ was used as a positive control.

Western Blot Analysis iNOS protein, COX-2 protein, $\mathrm{NF}-\kappa \mathrm{B}$ associated proteins, including nuclear p65, cytoplasmic $\mathrm{p} 65, \mathrm{p}-\mathrm{I} \kappa \mathrm{B}-\alpha$, and $\mathrm{I} \kappa \mathrm{B}-\alpha$, and MAPK associated proteins, including p-p38, p38, p-JNK, JNK, p-ERK, and ERK, were detected by Western blot analysis. Cells were lysed after harvest: the cells for the detection of iNOS and COX-2 were lysed using RIPA buffer, the cells for the detection of MAPK associated proteins were lysed using RIPA buffer and protease and phosphatase inhibitors, and the cells for NF- $\kappa$ B associated proteins were prepared using the nuclear extraction kit. After preparation, the samples were normalized for equal amounts of protein using the Bradford protein assay. Thereafter, $30 \mu \mathrm{g}$ of protein was loaded for each sample, separated on $7.5 \%$ (for proteins of iNOS, COX-2, and p65) or $12 \%$ (for proteins of MAPKs, and $\mathrm{I} \kappa \mathrm{B}-\alpha$ ) sodium dodecyl sulfate-polyacrylamide gel electrophoresis gels, and transferred to nitrocellulose membranes. This was followed by incubation with 5\% skim milk for $1 \mathrm{~h}$ at $25^{\circ} \mathrm{C}$, incubation with primary antibodies at $4{ }^{\circ} \mathrm{C}$ overnight, and incubation with secondary antibodies for $2 \mathrm{~h}$ at $25^{\circ} \mathrm{C}$. After each step, the membranes were washed by Tris-Buffered Saline-Tween 20 detergent (TBS-T). The protein bands were detected by enhanced chemiluminescence using Amersham ECL Prime Western blotting Detection Reagent.

Statistical Analysis Data were expressed as the mean \pm standard deviation (S.D.), by at least three independent experiments. One-way ANOVA and Tukey's multiple comparison test were used, performed by GraphPad Prism software, version 3.03 (GraphPad Software Inc., San Diego, CA, U.S.A.).

\section{RESULTS}

HPLC Analysis To determine which herbs mainly contributed to the SST formula, the samples of SST and seven ingredients were analyzed respectively by HPLC, and the chromatograms were shown in Fig. 1. Compared by the relative areas, the top five peaks were separately from Scutellariae Radix, Scutellariae Radix, Coptidis Rhizoma, Paeoniae Radix, and Coptidis Rhizoma, and their relative area was $26.1,8.8,4.0,3.2,2.8 \%$, respectively. Besides, the top peak was from the compound baicalin, which is an active substance of Scutellariae Radix.

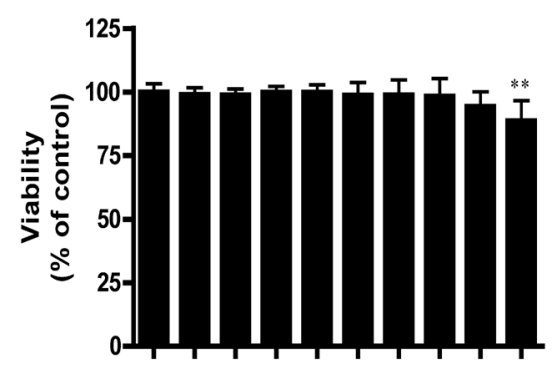

(b) $\operatorname{SST}(\mu \mathrm{g} / \mathrm{mL}) \quad-0.78 \quad 1.563 .136 .2512 .5 \quad 25 \quad 50100200$

Fig. 2. Effects of SST on the Viability of RAW264.7 Macrophages (a) and BV2 Microglial Cells (b)

Data shown represent the mean \pm standard deviation (S.D.) of three independent experiments. One-way ANOVA and Tukey's multiple comparison test were used. $* * p<0.01$ and $* * * p<0.001$, as compared to the control group. 
(a)

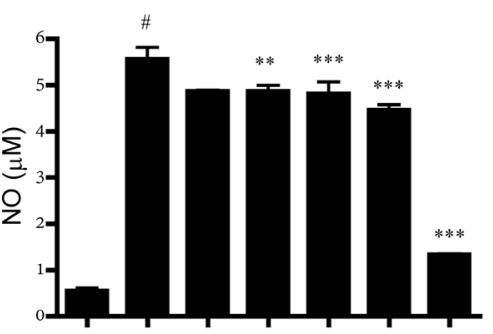

LPS $(1 \mu \mathrm{g} / \mathrm{mL})$

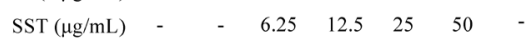

Butein $(10 \mu \mathrm{M})$

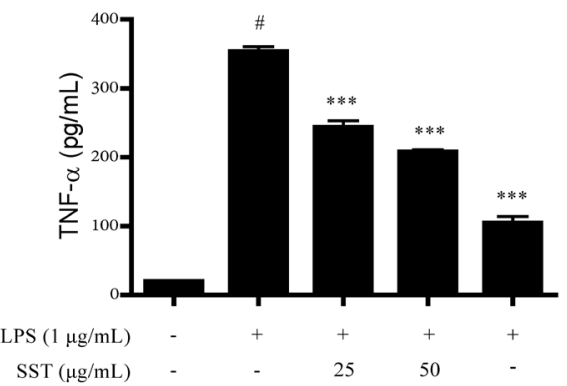

(c)

(d)

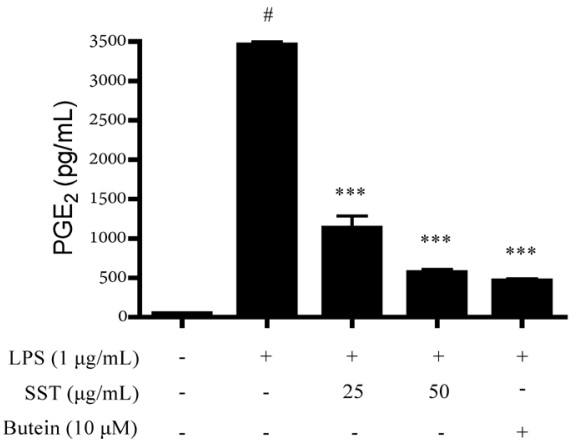

(b)

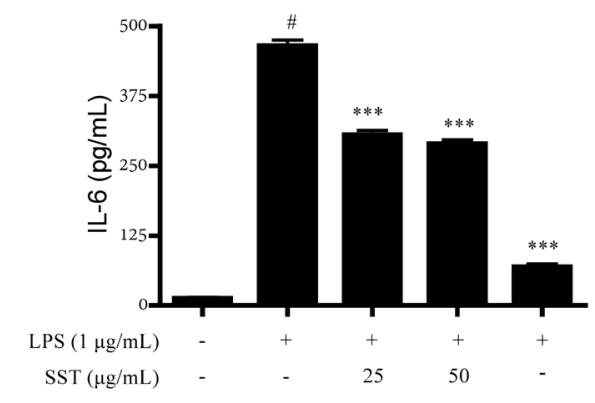

Fig. 3. Effects of SST on NO (a), PGE 2 (b), TNF- $\alpha$ (c), and IL-6 (d) in LPS-Stimulated RAW264.7 Macrophages

Butein $(10 \mu \mathrm{M})$ was used as a positive control. Data shown represent the mean \pm S.D. of three independent experiments. One-way ANOVA and Tukey's multiple comparison test were used. ${ }^{\#} p<0.001$, as compared to the control group; $* * p<0.01$ and ${ }^{* * *} p<0.001$, as compared to the LPS group.

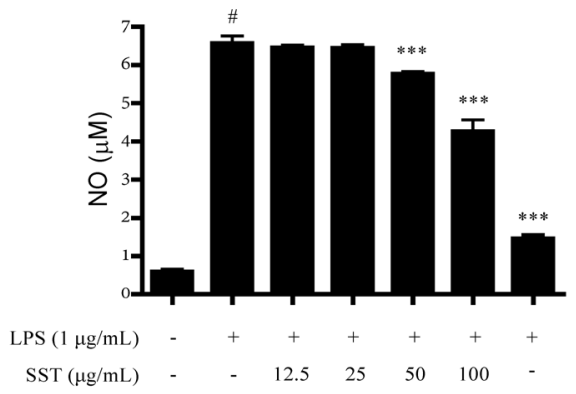

(a)
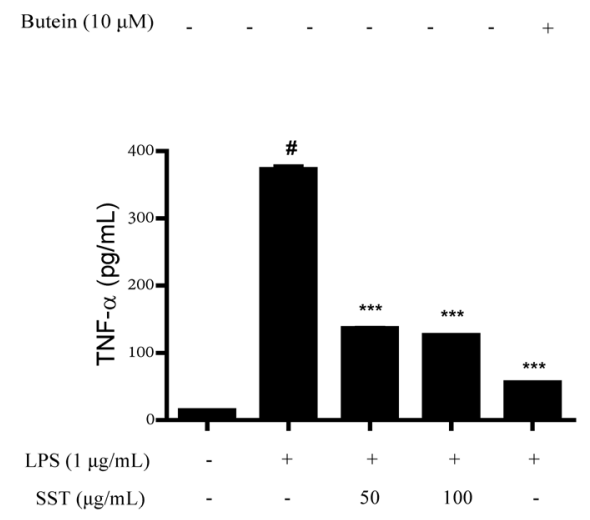

(c)

LPS $(1 \mu \mathrm{g} / \mathrm{mL})$

$\mathrm{SST}(\mu \mathrm{g} / \mathrm{mL})$

Butein $(10 \mu \mathrm{M})$

(d)

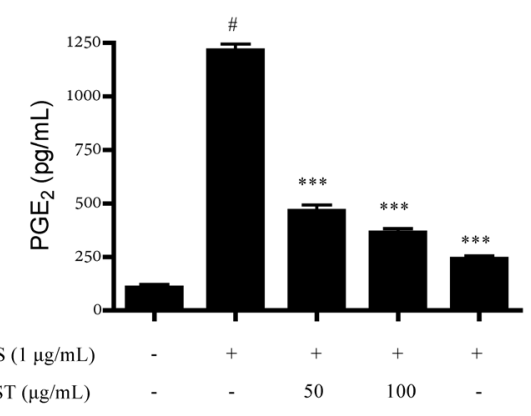

Butein $(10 \mu \mathrm{M})$

Fig. 4. Effects of SST on NO (a), PGE 2 (b), TNF- $\alpha$ (c), and IL-6 (d) in LPS-Stimulated BV2 Microglial Cells

Butein $(10 \mu \mathrm{M})$ was used as a positive control. Data shown represent the mean \pm S.D. of three independent experiments. One-way ANOVA and Tukey's multiple comparison test were used. ${ }^{\#} p<0.001$, as compared to the control group; $* p<0.01$ and $* * * p<0.001$, as compared to the LPS group. 
Cell Viability To evaluate the cytotoxicity of SST, we determined the cell viability of RAW264.7 macrophages and BV2 microglial cells following treatment with SST for $24 \mathrm{~h}$ at concentrations ranging from 0.78 to $200 \mu \mathrm{g} / \mathrm{mL}$. The results showed that SST concentration up to $50 \mu \mathrm{g} / \mathrm{mL}$ for RAW264.7 macrophages while $100 \mu \mathrm{g} / \mathrm{mL}$ for BV2 microglial cells had no effects on the viability (Fig. 2).

Effects of SST on the Production of NO, PGE 2 , TNF- $\alpha$, and IL-6 in LPS-Stimulated RAW264.7 Macrophages and BV2 Microglial Cells First, we evaluated the effective concentrations of SST by testing NO production in LPS-stimulated RAW264.7 macrophages. Cells were pre-treated with SST at $6.25,12.5,25$, and $50 \mu \mathrm{g} / \mathrm{mL}$ for $4 \mathrm{~h}$ and then stimulated with LPS $(1 \mu \mathrm{g} / \mathrm{mL})$ for $24 \mathrm{~h}$. SST markedly inhibited the production of NO at $12.5(* * p<0.01), 25$ and $50(* * * p<0.001)$ $\mu \mathrm{g} / \mathrm{mL}$ (Fig. 3). Subsequent experiments were conducted with SST at 25 and $50 \mu \mathrm{g} / \mathrm{mL}$ in RAW264.7 macrophages. Also, we evaluated the effective concentrations of SST by testing NO production in LPS-stimulated BV2 microglial cells. Cells were pre-treated with SST at $12.5,25,50$, and $100 \mu \mathrm{g} / \mathrm{mL}$ for $4 \mathrm{~h}$ and then stimulated with LPS $(1 \mu \mathrm{g} / \mathrm{mL})$ for $24 \mathrm{~h}$. SST markedly inhibited the production of $\mathrm{NO}$ at 50 and $100 \mu \mathrm{g} / \mathrm{mL}$
$(* * * p<0.001)$ (Fig. 4). Subsequent experiments were conducted with SST at 50 and $100 \mu \mathrm{g} / \mathrm{mL}$ in BV2 microglial cells. To investigate if SST would inhibit LPS-induced increases in the secretion of inflammatory mediators, we evaluated the production of PGE 2 , TNF- $\alpha$, and IL-6. Cells were pre-treated with SST for $4 \mathrm{~h}$ and then stimulated with LPS $(1 \mu \mathrm{g} / \mathrm{mL})$ for $24 \mathrm{~h}$. Pre-treatment with SST inhibited the LPS-induced production of PGE 2 , TNF- $\alpha$, and IL-6, in a dose-dependent manner, in LPS-stimulated RAW264.7 macrophages (Fig. 3) as well as BV2 microglial cells (Fig. 4).

Effects of SST on the Expression of iNOS and COX-2 Proteins in LPS-Stimulated RAW264.7 Macrophages and BV2 Microglial Cells We investigated the effects of SST on LPS-induced upregulation of iNOS and COX-2 proteins in LPS-stimulated RAW264.7 macrophages and BV2 microglial cells. Cells were pre-treated with SST for $4 \mathrm{~h}$ and then stimulated with LPS $(1 \mu \mathrm{g} / \mathrm{mL})$ for $18 \mathrm{~h}$. The expression of iNOS and COX-2 proteins were increased by LPS but decreased by pre-treatment with SST in both LPS-stimulated RAW264.7 macrophages (Fig. 5) and BV2 microglial cells (Fig. 6), which were consistent with the effects on the production of NO and $\mathrm{PGE}_{2}$, respectively (Figs. 3, 4).
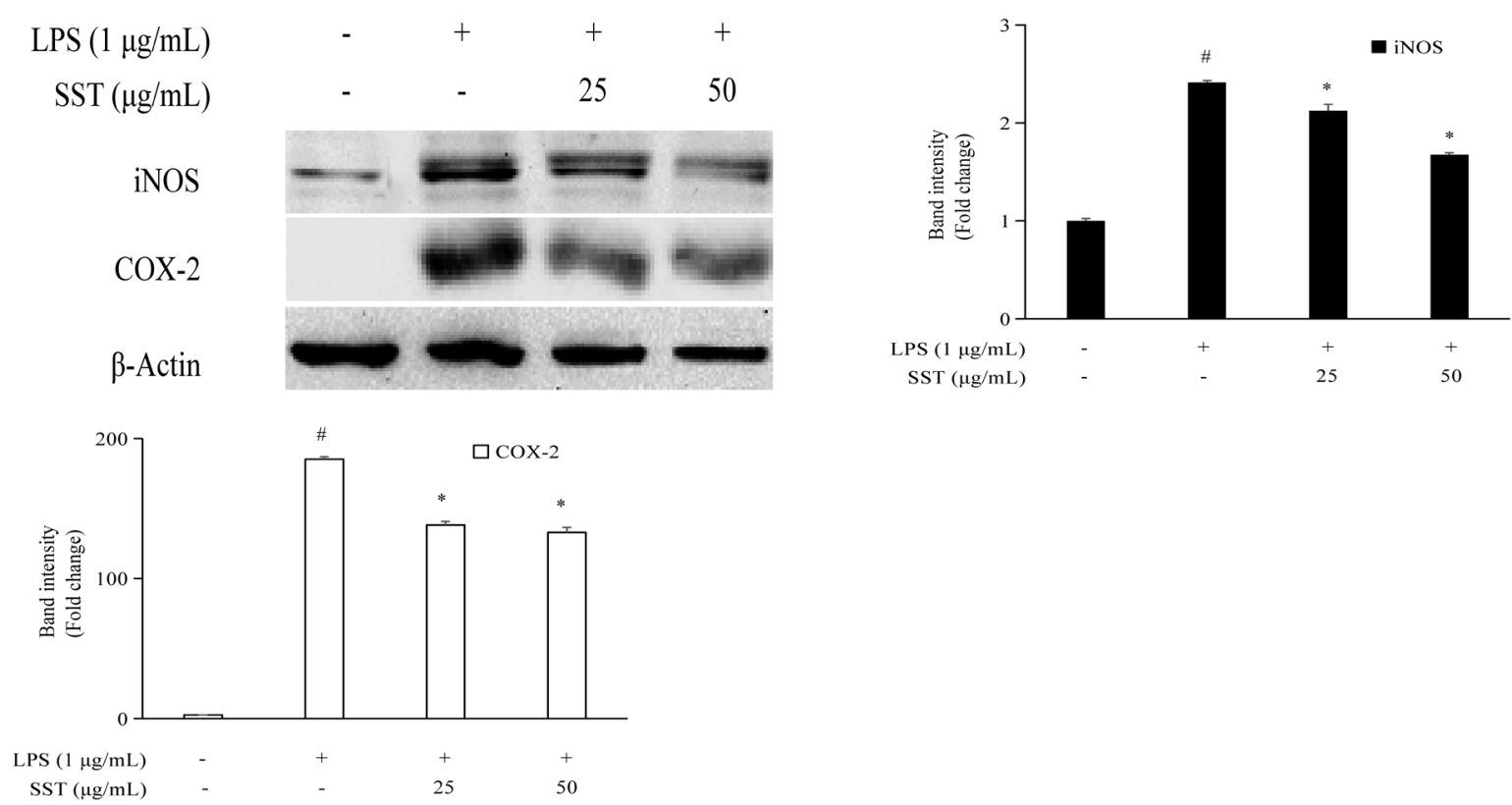

Fig. 5. Effect of SST on Expression of iNOS and COX-2 Proteins in LPS-Stimulated RAW264.7 Macrophages

The band intensity was quantified by densitometry and normalized to the intensity of the $\beta$-actin. Data shown represent the mean \pm S.D. of three independent experiments. One-way ANOVA and Tukey's multiple comparison test were used. ${ }^{*} p<0.05$, as compared to the control group; * $p<0.05$, as compared to the LPS group.
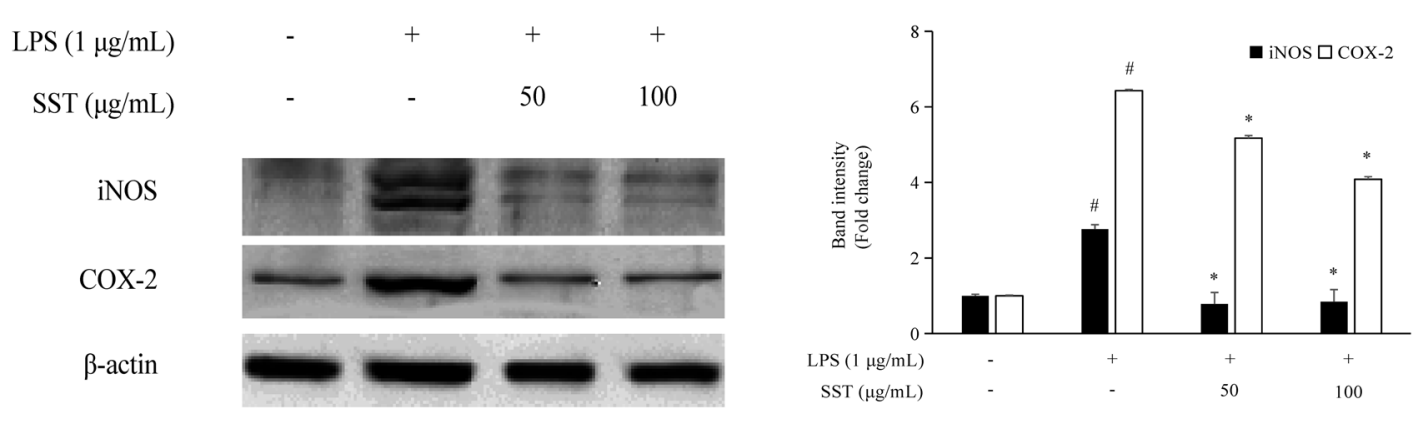

Fig. 6. Effect of SST on Expression of iNOS and COX-2 Proteins in LPS-Stimulated BV2 Microglial Cells

The band intensity was quantified by densitometry and normalized to the intensity of the $\beta$-actin. Data shown represent the mean \pm S.D. of three independent experiments. One-way ANOVA and Tukey's multiple comparison test were used. ${ }^{*} p<0.05$, as compared to the control group; ${ }^{*} p<0.05$, as compared to the LPS group. 

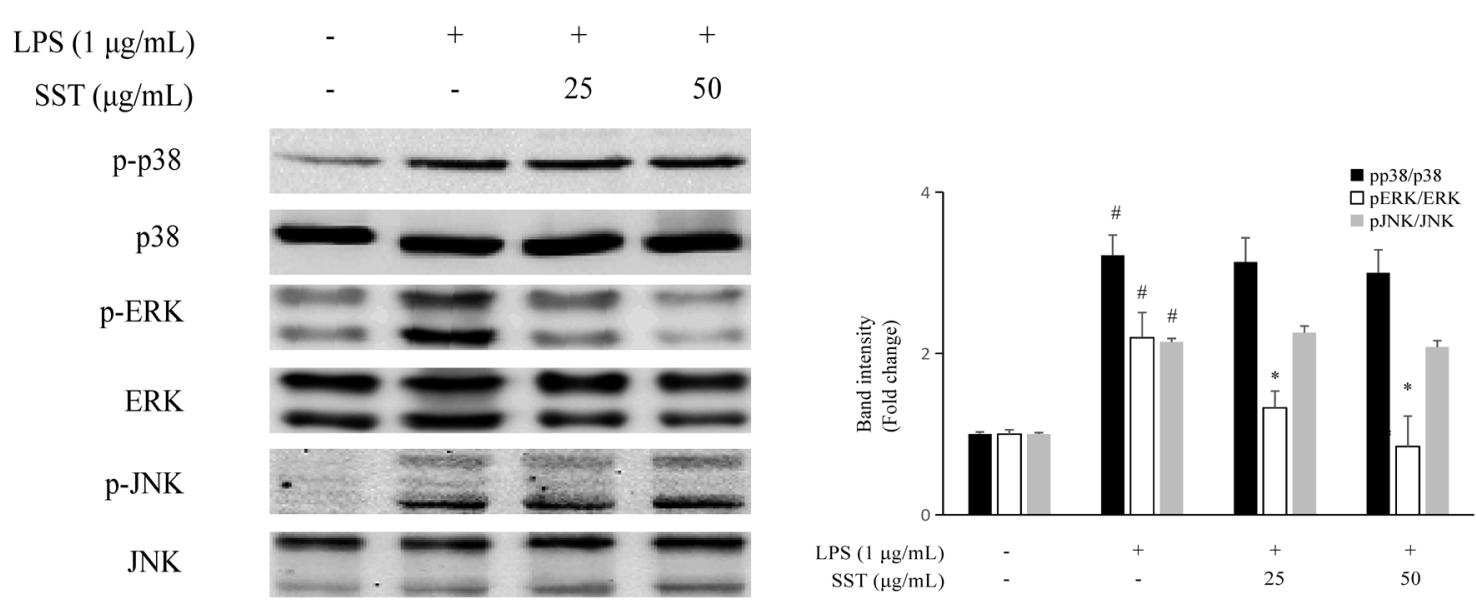

Fig. 7. Effects of SST on the MAPK Pathways, Including Expression of p-p38, p38, p-ERK, ERK, p-JNK, and JNK in LPS-Stimulated RAW264.7 Macrophages

The band intensity was quantified by densitometry and normalized. Data shown represent the mean \pm S.D. of three independent experiments. One-way ANOVA and Tukey's multiple comparison test were used. ${ }^{\#} p<0.05$, as compared to the control group; ${ }^{*} p<0.05$, as compared to the LPS group.

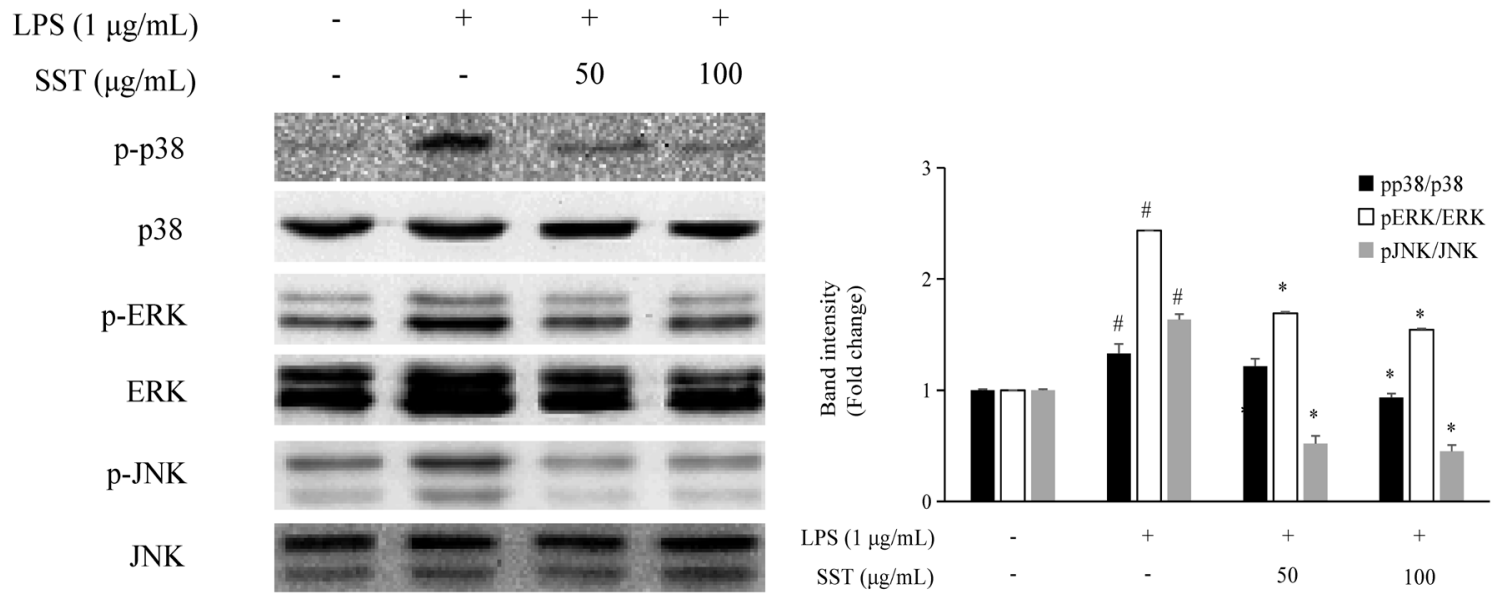

Fig. 8. Effects of SST on the MAPK Pathways, Including Expression of p-p38, p38, p-ERK, ERK, p-JNK, and JNK in LPS-Stimulated BV2 Microglial Cells

The band intensity was quantified by densitometry and normalized. Data shown represent the mean \pm S.D. of three independent experiments. One-way ANOVA and Tukey's multiple comparison test were used. ${ }^{*} p<0.05$, as compared to the control group; ${ }^{*} p<0.05$, as compared to the LPS group.

Effects of SST on the MAPK Pathway in LPS-Stimulated RAW264.7 Macrophages and BV2 Microglial Cells We examined if the anti-inflammatory effects of SST would contribute to the activation of the MAPK pathway in LPS-stimulated RAW264.7 macrophages and BV2 microglial cells. Cells were pre-treated with SST for $4 \mathrm{~h}$ and then stimulated with LPS $(1 \mu \mathrm{g} / \mathrm{mL})$ for $1 \mathrm{~h}$. As shown, LPS markedly increased the phosphorylation of p38, JNK, and ERK in both cells. In LPSstimulated RAW264.7 macrophages, pre-treatment with SST only significantly inhibited the activation of the ERK pathway (Fig. 7). However, in LPS-stimulated BV2 microglial cells, pre-treatment with SST significantly inhibited the activation of all the p38, ERK, and JNK pathways (Fig. 8).

Effects of SST on the NF- $\kappa$ B Pathway in LPS-Stimulated RAW264.7 Macrophages and BV2 Microglial Cells We also investigated if the anti-inflammatory effects of SST would contribute to the activation of the NF- $\kappa \mathrm{B}$ pathway in LPS-stimulated RAW264.7 macrophages and BV2 microglial cells. Cells were pre-treated with SST for $4 \mathrm{~h}$ and then stimulated with LPS $(1 \mu \mathrm{g} / \mathrm{mL})$ for $0.5 \mathrm{~h}$. After LPS stimulation, the phosphorylation and degradation of $\mathrm{I} \kappa \mathrm{B}-\alpha$ increased, and $\mathrm{p} 65$ in cytoplasm decreased while translocating into the nucleus. However, these changes in activation were significantly inhibited by SST pre-treatment in both LPS-stimulated RAW264.7 macrophages (Fig. 9) and BV2 microglial cells (Fig. 10).

\section{DISCUSSION}

SST formula has been widely used for the treatment of various gynecological diseases. ${ }^{1-3)}$ The formula consists of seven herbs, and some of the ingredients have been reported to have anti-inflammatory or anti-neuroinflammatory effects. ${ }^{4-7)}$ To expand the clinical use and explore new functions of SST, we evaluated the anti-inflammatory or anti-neuroinflammatory effects of SST, which have not been studied yet. As we know, inflammation is a kind of defense mechanism initiated by the body's immune system. It is a process that involves the action of multiple factors that would happen in various diseases, including gynecological diseases and nervous system diseases. ${ }^{18)}$ Thus, it is necessary and essential to inhibit inflammation during treatment for these diseases.

Our study demonstrated that SST herbal formula showed 


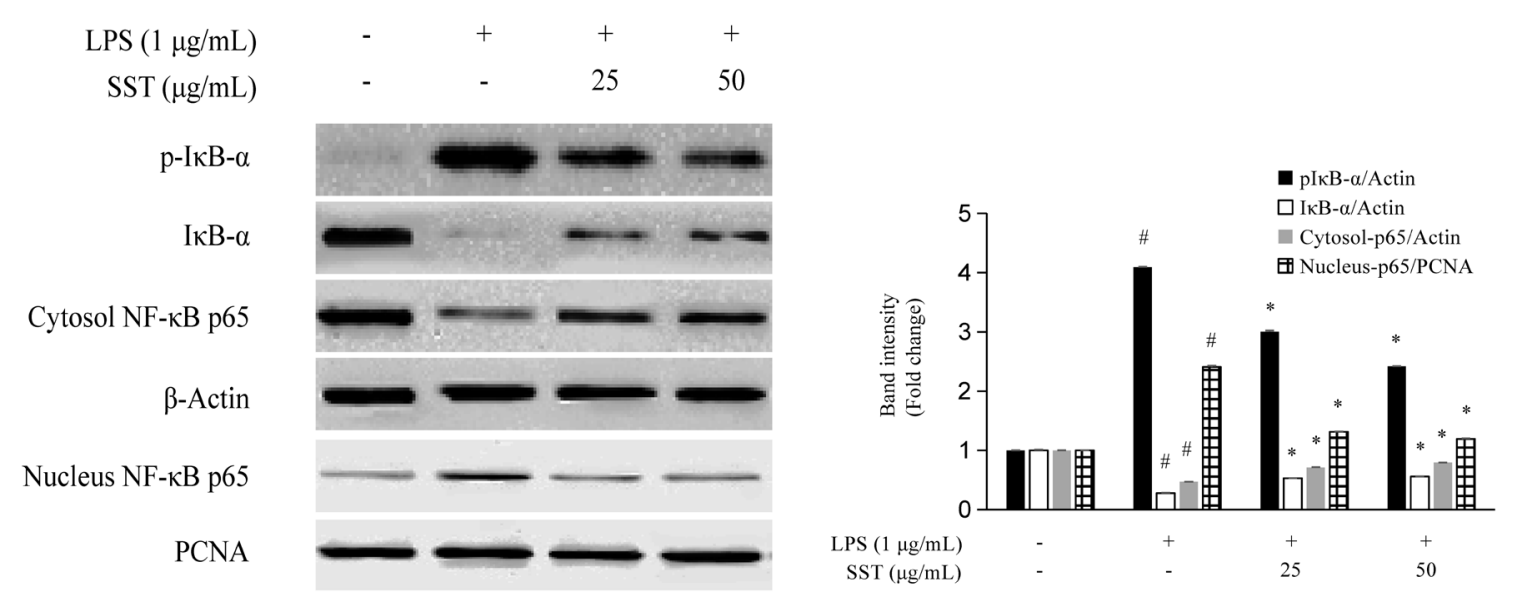

Fig. 9. Effects of SST on the NF- $\kappa$ B Pathways Including Expression of p65, p-I $\kappa \mathrm{B}-\alpha$, and I $\kappa \mathrm{B}-\alpha$ in LPS-Stimulated RAW264.7 Macrophages

The band intensity was quantified by densitometry and normalized to the intensity of the $\beta$-actin or PCNA band. Data shown represent the mean \pm S.D. of three independent experiments. One-way ANOVA and Tukey's multiple comparison test were used. ${ }^{*} p<0.05$, as compared to the control group; $* p<0.05$, as compared to the LPS group.

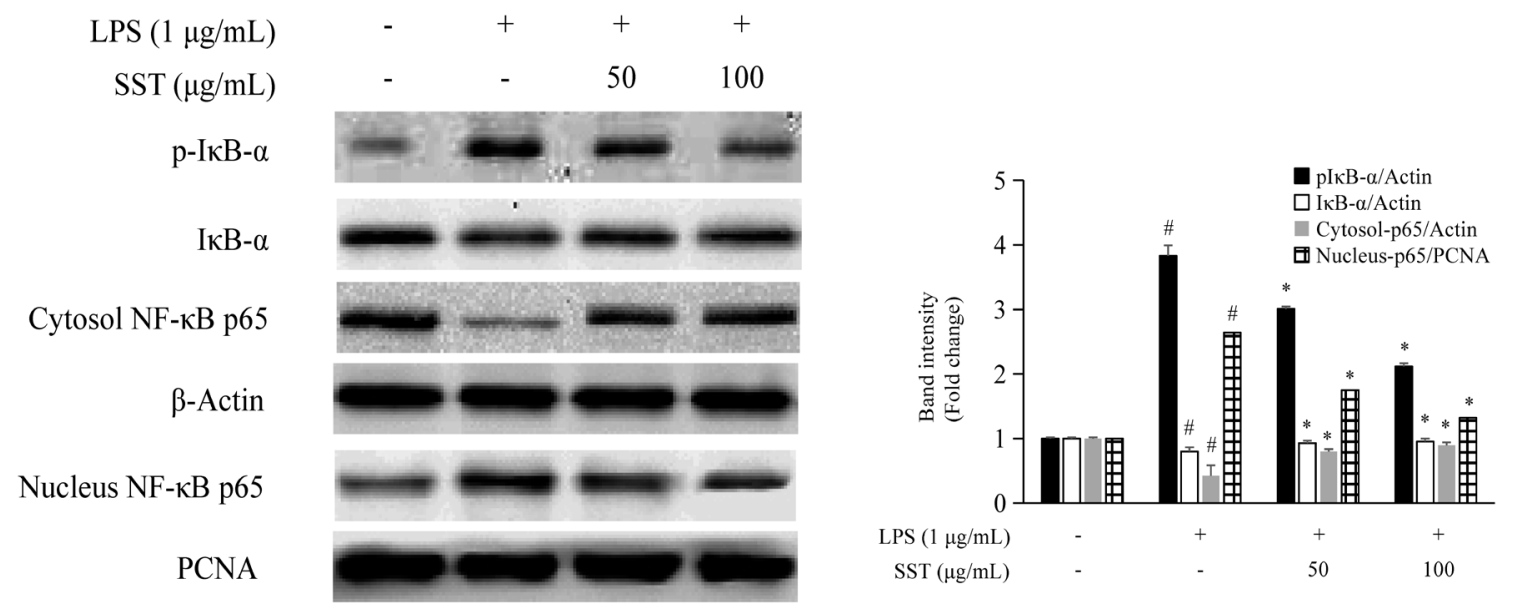

Fig. 10. Effects of SST on the NF- $\kappa$ B Pathways, Including Expression of p65, p-I $\kappa$ B- $\alpha$, and I $\kappa$ B- $\alpha$ in LPS-Stimulated BV2 Microglial Cells

The band intensity was quantified by densitometry and normalized to the intensity of the $\beta$-actin or PCNA band. Data shown represent the mean \pm S.D. of three independent experiments. One-way ANOVA and Tukey's multiple comparison test were used. ${ }^{*} p<0.05$, as compared to the control group; ${ }^{*} p<0.05$, as compared to the LPS group.

anti-inflammatory effects in LPS-stimulated RAW264.7 macrophages and BV2 microglial cells. Macrophages and microglia are major inflammatory cells. Macrophages play a crucial role in developing the inflammatory response, and during the process of inflammation, macrophages are activated to secrete various pro-inflammatory mediators and cytokines. ${ }^{19)}$ Microglial cells are considered to be the macrophages in the CNS. It is suggested that abnormally activated microglia cells induce the excessive production of pro-inflammatory mediators, resulting in neuroinflammation or neurodegenerative diseases. ${ }^{20)}$ Therefore, interventions to prevent macrophages or microglial activation would be promising therapeutic strategies for the prevention or treatment of inflammatory or neuroinflammatory disorders. The models utilizing LPS-stimulated RAW264.7 macrophages and BV2 microglial cells have been frequently in the course of research for anti-inflammatory or anti-neuroinflammatory effects employed. ${ }^{9,12)}$ LPS, as an exogenous bacterial endotoxin, could activate both macrophages and microglial, and these activations would lead to the release of pro-inflammatory mediators including NO, PGE, IL-6, and TNF- $\alpha^{21,22)} \mathrm{NO}$ is derived from nitric oxide synthases (NOS), and iNOS is one form of NOS induced by LPS. PGE2 is also an essential mediator of inflammatory symptoms and is mainly formed through the activity of the COX-2 enzyme. All of these are involved in the pathogenesis of various inflammatory as well as neuroinflammatory pathophysiological conditions. ${ }^{23-25)}$ Thus, it is suggested that inhibitors of these mediators are useful in the treatment of inflammation-related diseases. ${ }^{26)}$ Pro-inflammatory cytokines, including IL-6 and TNF- $\alpha$, play a crucial role in inflammation and host defense and mediate neurodegeneration in CNS immune responses. ${ }^{27,28)}$ According to our results, SST exerted anti-inflammatory effects in LPS-stimulated RAW264.7 macrophages and anti-neuroinflammatory effects in LPSstimulated BV2 microglial cells by inhibiting the production of LPS-induced pro-inflammatory mediators including NO, PGE2, iNOS, COX-2, IL-6, and TNF- $\alpha$. However, the concentrations in SST pre-treated RAW264.7 macrophages and BV2 microglial cells were different. It contributed to different cell toxicity of SST between the two cells, and according to our data, SST has lower toxicity in BV2 microglial cells than in RAW264.7 macrophages.

After LPS binds to the Toll-like receptor 4 (TLR4) of macrophages and microglia, it generates a variety of pathways of inflammatory processes. ${ }^{29)}$ Activated TLR4 transfers through two main pathways, including TLR-mediated myeloid dif- 
ferentiation factor 88 (MyD88)-dependent pathway and the Toll/IL-1 receptor domain-containing adaptor induction of the interferon- $\beta$ (TRIF)-dependent pathway. ${ }^{30)}$ MyD88 is an adapter protein that would lead to the activation of NF- $\kappa \mathrm{B}$ and MAPKs. ${ }^{31)}$ Therefore, in this study, we investigated the effects of SST on LPS-stimulated NF- $\kappa$ B and MAPKs activation. MAPKs are composed of three major subunits, p38, ERK, and JNK, and are a family of serine/threonine protein kinases related to the activation of inflammatory responses, including the production of inflammatory mediators such as iNOS, COX-2, ILs, and TNF- $\alpha{ }^{32-36)}$ Our results showed that SST inhibited the phosphorylation of ERK in LPS-stimulated RAW264.7 macrophages and decreased the phosphorylation of p38, ERK, and JNK in LPS-stimulated BV2 microglial cells. It demonstrated that MAPKs pathways could be targets of SST treatment. However, ERK could be a common pathway for both cells, and p38 and JNK could be the additional pathways for BV2 microglial cells. NF- $\kappa \mathrm{B}$ is a critical transcription factor that regulates inflammatory responses. In the inactive state, NF- $\kappa \mathrm{B}$ exists in the cytoplasm and complexes with the inhibitor of NF- $\kappa \mathrm{B}(\mathrm{I} \kappa \mathrm{B}) .{ }^{37)}$ Upon activation by stimulation with LPS, NF- $\kappa \mathrm{B}$ can be activated following the phosphorylation and degradation of $\mathrm{I} \kappa \mathrm{B}-\alpha$ in the cytosol and increased translocation of p65 from the cytosol into the nucleus. ${ }^{38)}$ These responses lead to the transcription of pro-inflammatory cytokines, chemokines, and adhesion molecules, including NO, PGE2, iNOS, COX-2, IL- 6 , and TNF- $\alpha{ }^{39,40)}$ Thus, NF- $\kappa$ B has been regarded as the molecular target for the treatment of inflammation-related and neuroinflammation-related diseases. In our study, SST inactivated the phosphorylation and degradation of $\mathrm{I} \kappa \mathrm{B}-\alpha$ and the nuclear translocation of $\mathrm{p} 65$ in both RAW264.7 macrophages and BV2 microglial cells. It demonstrated that SST could exhibit anti-inflammatory effects through the NF- $\kappa$ B pathway in both RAW264.7 macrophages and BV2 microglial cells.

Furthermore, according to the HPLC analysis, the major components of SST water extract were from Scutellariae Radix, Coptidis Rhizoma, and Paeoniae Radix. Also, the most abundant substance was baicalin, which was from Scutellariae Radix. In another way, baicalin would be a marker or for quality control for SST water extract. However, since SST is a formula, it is difficult to say whether baicalin is the most effective substance or just one component of SST contributing to its anti-inflammatory and anti-neuroinflammatory effects.

In summary, SST exhibited anti-inflammatory effects through MAPKs and NF- $\kappa$ B pathways in both LPS-stimulated RAW264.7 macrophages and BV2 microglial cells, and these effects may be correlated mainly with Scutellariae Radix, Coptidis Rhizoma, and Paeoniae Radix. It demonstrated that SST is a potential therapeutic formula for inflammation or neuroinflammation and neurodegenerative diseases.

Acknowledgments This research was supported by the National Research Foundation of Korea (NRF) Grants funded by the Korea government (NRF-2017R1A5A2015805).

Conflict of Interest The authors declare no conflict of interest.

Supplementary Materials The online version of this article contains supplementary materials.

\section{REFERENCES}

1) The Golden Mirror in the imperial court of the Qianlong emperor, Vol. 44 (1739-1742).

2) Tang JL. 80 cases for preceeded menorrhea treatment of Sanghuang-Siwu-Tang. Henan Traditional Chinese Medicine, 29, 588-589 (2009).

3) Song ZL. Modified Sanhuang Siwu Decoction in treating adolescent functional uterine bleeding. Sichuan Medicine, 1, 109 (1980).

4) Hsiao G, Chen YC, Lin JH, Lin KH, Chou DS, Lin CH, Sheu JR. Inhibitory mechanisms of tetramethylpyrazine in middle cerebral artery occlusion (MCAO)-induced focal cerebral ischemia in rats. Planta Med., 72, 411-417 (2006).

5) Zhang HR, Peng JH, Cheng XB, Shi BZ, Zhang MY, Xu RX. Paeoniflorin attenuates amyloidogenesis and the inflammatory responses in a transgenic mouse model of Alzheimer's disease. Neurochem. Res., 40, 1583-1592 (2015).

6) Wang ZY, Yang Y, Liu MX, Wei Y, Liu JG, Pei H, Li H. Rhizoma Coptidis for Alzheimer's disease and vascular dementia: a literature review. Curr. Vasc. Pharmacol., 18, 358-368 (2020).

7) Yan JJ, Du GH, Qin XM, Gao L. Baicalein attenuates the neuroinflammation in LPS-activated BV-2 microglial cells through suppression of pro-inflammatory cytokines, COX $2 / \mathrm{NF}-\kappa \mathrm{B}$ expressions and regulation of metabolic abnormality. Int. Immunopharmacol., 79, 106092 (2020).

8) Parameswaran N, Patial S. Tumor necrosis factor- $\alpha$ signaling in macrophages. Crit. Rev. Eukaryot. Gene Expr., 20, 87-103 (2010).

9) Heller RA, Schena M, Chai A, Shalon D, Bedilion T, Gilmore J, Woolley DE, Davis RW. Discovery and analysis of inflammatory disease-related genes using cDNA microarrays. Proc. Natl. Acad. Sci. U.S.A., 94, 2150-2155 (1997).

10) Groh J, Martini R. Neuroinflammation as modifier of genetically caused neurological disorders of the central nervous system: understanding pathogenesis and chances for treatment. Glia, 65, 1407-1422 (2017)

11) Cianciulli A, Calvello R, Porro C, Trotta $T$, Salvatore R, Panaro MA. PI3k/Akt signaling pathway plays a crucial role in the antiinflammatory effects of curcumin in LPS-activated microglia. Int. Immunopharmacol., 36, 282-290 (2016).

12) Henn A, Lund S, Hedtjärn M, Schrattenholz A, Pörzgen P, Leist M. The suitability of BV2 cells as alternative model system for primary microglia cultures or for animal experiments examining brain inflammation. $A L T E X, 26,83-94$ (2009).

13) Dai JN, Zong Y, Zhong LM, Li YM, Zhang W, Bian LG, Ai QL, Liu YD, Sun J, Lu D. Gastrodin inhibits expression of inducible NO synthase, cyclooxygenase-2 and proinflammatory cytokines in cultured LPS-stimulated microglia via MAPK pathways. PLoS ONE, 6, e21891 (2011).

14) $\mathrm{Li}$ CQ, He LC, Jin JQ. Atractylenolide I and atractylenolide III inhibit lipopolysaccharide-induced TNF- $\alpha$ and NO production in macrophages. Phytother. Res., 21, 347-353 (2007).

15) Guo C, Yang L, Wan CX, Xia YZ, Zhang C, Chen MH, Wang ZD, Li ZR, Li XM, Geng YD, Kong LY. Anti-neuroinflammatory effect of Sophoraflavanone $\mathrm{G}$ from Sophora alopecuroides in LPSactivated $\mathrm{BV}_{2}$ microglia by MAPK, JAK/STAT and Nrf2/HO-1 signaling pathways. Phytomedicine, 23, 1629-1637 (2016).

16) Yuan L, Wu Y, Ren X, Liu Q, Wang J, Liu X. Isoorientin attenuates lipopolysaccharide-induced pro-inflammatory responses through down-regulation of ROS-related MAPK/NF $\kappa \mathrm{B}$ signaling pathway in BV-2 microglia. Mol. Cell. Biochem., 386, 153-165 (2014).

17) На TM, Ko W, Lee SJ, Kim YC, Son JY, Sohn JH, Yim JH, Oh H. Anti-inflammatory effects of curvularin-type metabolites from a marine-derived fungal strain Penicillium sp. SF-5859 in lipopolysaccharide-induced RAW264.7 macrophages. Mar. Drugs, 15, 282 (2017).

18) Kiem PV, Cuong LC, Tai BH, Nhiem NX, Anh HL, Quang TH, 
Ngan NT, Oh H, Kim YC. New lignans from antidesma hainanensis inhibit $\mathrm{NO}$ production in $\mathrm{BV}_{2}$ microglial cells. Chem. Pharm. Bull. 64, 1707-1712 (2016).

19) Fujiwara N, Kobayashi K. Macrophages in inflammation. Curr. Drug Targets Inflamm. Allergy, 4, 281-286 (2005).

20) Stephenson J, Nutma E, van der Valk P, Amor S. Inflammation in CNS neurodegenerative diseases. Immunology, 154, 204-219 (2018).

21) Lull ME, Block ML. Microglial activation and chronic neurodegeneration. Neurotherapeutics, 7, 354-365 (2010).

22) Berenbaum F. Proinflammatory cytokines, prostaglandins, and the chondrocyte: mechanisms of intracellular activation. Joint Bone Spine, 67, 561-564 (2000).

23) Vane JR, Mitchell JA, Appleton I, Tomlinson A, Bishop-Bailey D, Croxtall J, Willoughby DA. Inducible isoforms of cyclooxygenase and nitric-oxide synthase in inflammation. Proc. Natl. Acad. Sci. U.S.A., 91, 2046-2050 (1994).

24) Samuelsson B. Leukotrienes: mediators of immediate hypersensitivity reactions and inflammation. Science, 220, 568-575 (1983).

25) Lowenstein CJ, Glatt CS, Bredt DS, Snyder SH. Cloned and expressed macrophage nitric oxide synthase contrasts with the brain enzyme. Proc. Natl. Acad. Sci. U.S.A., 89, 6711-6715 (1992).

26) Jeong SO, Oh GS, Ha HY, Soon Koo B, Sung Kim H, Kim YC, Kim EC, Lee KM, Chung HT, Pae HO. Dimethoxycurcumin, a synthetic curcumin analogue, induces heme oxygenase-1 expression through Nrf2 activation in RAW264.7 macrophages. J. Clin. Biochem. Nutr., 44, 79-84 (2009).

27) Kempuraj D, Thangavel R, Natteru PA, Selvakumar GP, Saeed D, Zahoor H, Zaheer S, Iyer SS, Zaheer A. Neuroinflammation induces neurodegeneration. J. Neurol. Neurosurg. Spine, 1, 1003 (2016).

28) Mircic M, Kavanaugh A. Inhibition of IL6 in rheumatoid arthritis and juvenile idiopathic arthritis. Exp. Cell Res., 317, 1286-1292 (2011).

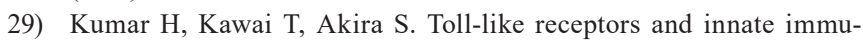
nity. Biochem. Biophys. Res. Commun., 388, 621-625 (2009).

30) Noman AS, Koide N, Khuda II, Dagvadorj J, Tumurkhuu G, Naiki Y, Komatsu T, Yoshida T, Yokochi T. Thalidomide inhibits lipopolysaccharide-induced nitric oxide production and prevents lipopolysaccharide-mediated lethality in mice. FEMS Immunol.
Med. Microbiol., 56, 204-211 (2009).

31) Cho KH, Kim DC, Yoon CS, Ko WM, Lee SJ, Sohn JH, Jang JH, Ahn JS, Kim YC, Oh H. Anti-neuroinflammatory effects of citreohybridonol involving TLR4-MyD88-mediated inhibition of NF-кB and MAPK signaling pathways in lipopolysaccharide-stimulated $\mathrm{BV}_{2}$ cells. Neurochem. Int., 95, 55-62 (2016).

32) González-Scarano F, Baltuch G. Microglial as mediators of inflammatory and degenerative diseases. Annu. Rev. Neurosci., 22, 219-240 (1999).

33) Lee S, Kim DC, Baek HY, Lee KD, Kim YC, Oh H. Antineuroinflammatory effects of tryptanthrin from Polygonum tinctorium Lour. in lipopolysaccharide-stimulated $\mathrm{BV}_{2}$ microglial cells. Arch. Pharm. Res., 41, 419-430 (2018).

34) Kim KS, Lee DS, Kim DC, Yoon CS, Ko W, Oh H, Kim YC. Antiinflammatory effects and mechanisms of action of coussaric and betulinic acids isolated from Diospyros kaki in lipopolysaccharidestimulated RAW 264.7 macrophages. Molecules, 21, 1206 (2016).

35) Agostinho P, Cunha RA, Oliveira C. Neuroinflammation, oxidative stress and the pathogenesis of Alzheimer's disease. Curr. Pharm. Des., 16, 2766-2778 (2010).

36) Nathan C. Points of control in inflammation. Nature, 420, 846-852 (2002).

37) Chen CC. Signal transduction pathways of inflammatory gene expressions and therapeutic implications. Curr. Pharm. Des., 12, 3497-3508 (2006).

38) Velagapudi R, Aderogba M, Olajide OA. Tiliroside, a dietary glycosidic flavonoid, inhibits TRAF-6/NF- $\kappa \mathrm{B} / \mathrm{p} 38$-mediated neuroinflammation in activated BV2 microglia. Biochim. Biophys. Acta, 1840, 3311-3319 (2014).

39) Im EJ, Kim SJ, Hong SB, Park JK, Rhee MH. Anti-inflammatory activity of Bee Venom in BV2 microglial cells: mediation of MyD88-dependent NF- $\kappa \mathrm{B}$ signaling pathway. Evid. Based Complement. Alternat. Med., 2016, 3704764 (2016).

40) Lu MY, Chen CC, Lee LY, Lin TW, Kuo CF. $N^{6}$-(2-Hydroxyethyl) adenosine in the medicinal mushroom Cordyceps cicadae attenuates lipopolysaccharide-stimulated pro-inflammatory responses by suppressing TLR4-mediated NF- $\kappa$ B signaling pathways. J. Nat. Prod., 78, 2452-2460 (2015). 